\title{
Obesity in 7 - 10-year-old children in urban primary schools in Port Elizabeth
}

\author{
J McKersie, MA Human Movement Science (Biokinetics); M L Baard, DPhil \\ Department of Human Movement Science and Dietetics, Nelson Mandela Metropolitan University, Port Elizabeth, South Africa
}

Corresponding author: $M$ L Baard (maryna.baard@nmmu.ac.za)

\begin{abstract}
Objective. The primary aim of this study was to quantify the prevalence of overweight and obesity among urban 7 - 10-year-old children in affluent (quintile 5) English-medium primary schools in Port Elizabeth.

Method. A quantitative, descriptive one-way cross-sectional research design utilising random sampling was used. A once-off survey consisted of anthropometrical assessment of body mass index (BMI) according to standardised procedures. To classify children into weight categories, the International Obesity Task Force $z$-score BMI cut-off criteria were used. Post-hoc analysis consisted of one-way analysis of variance and $\chi^{2}$ tests. Level of significance was set at $p<0.05$. A total of 713 children participated in the study.

Results. Overweight prevalence was $20.9 \%(n=149)$ and obesity prevalence was $9.8 \%(n=70)$. A significant interaction was found for overweight and obesity levels by gender and age $(F=7.2, p=0.01)$. Of the boys $(N=372) 18.5 \%(n=69)$ were overweight and $6.9 \%(n=26)$ were obese. The girls $(N=341)$ had a $23.5 \%(n=80)$ overweight rate and $12,9 \%(n=44)$ were obese. The highest prevalence of overweight $(24.7 \%$, $n=43, N=174)$ was found in children aged 10 , and the highest prevalence of obesity $(12.5 \%, n=21, N=167)$ was found in children aged 8 .

Conclusion. Results highlighted the rising prevalence of overweight and obesity among urban children from economically privileged settings. Future research into paediatric obesity is needed to curb the growing incidence.
\end{abstract}

S Afr J SM 2014;26(2):55-58. DOI:10.7196/SAJSM.526

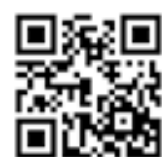

The prevalence of excess body weight is becoming a leading threat to the health of children, much more rapidly in developing countries than in industrialised ones. The World Health Organization (WHO) published a report stating that in $2011,75 \%$ of overweight children were from developing countries. ${ }^{[1]}$ This percentage accounts for 30 million children of 40 million worldwide. ${ }^{[1]}$ South Africa (SA) has the highest rate of obesity in sub-Saharan Africa and the prevalence has doubled from 1990 to 2010. ${ }^{[2]}$ The $S A$ Youth Risk Behaviour Survey, 2002 showed that the prevalence of overweight was over $17 \%$, with boys less at risk $(6,9 \%)$ than girls (25\%). ${ }^{[3]}$ Results from the Health of the Nation Study indicated that $31.9 \%$ of SA children aged 6 to 13 were overweight and $8.1 \%$ were obese. ${ }^{[4]}$ Overweight and obesity rates of boys were $14.0 \%$ and $3.2 \%$, respectively, while $17.9 \%$ of girls were rated as overweight and $4.9 \%$ as obese. ${ }^{[4]}$ Cognisance should be taken of the fact that the study was published 7 years ago. Research indicates that obesity in children tends to track into adulthood, unless interventions are put into place at a young age.${ }^{[5]}$ Literature reports that $70-80 \%$ of obese adolescents developed into obese adults. ${ }^{[6]}$

Associated with childhood obesity are increased risk of non-communicable diseases, decreased quality of life and premature death. ${ }^{[7]}$ According to the WHO estimates, by 2020 non-communicable diseases will account for almost three-quarters of all deaths in the developing world. ${ }^{[8]}$ Obese children are at risk for chronic disease and are prone to developing insulin resistance, metabolic syndrome, asthma and polycystic ovarian syndrome, and have an increased risk of orthopaedic complications. ${ }^{[6]}$ Given the tracking of obesity and associated risk factors, childhood is a key developmental period for early identification and prevention of excessive adiposity. ${ }^{[9]}$

To date, researchers in SA have focused mainly on quantifying undernutrition in rural African children from impoverished settings based on the nutrition transition perspective. ${ }^{[10,11]}$ SA schools are divided into five categories or quintiles, with the poorest schools in quintile 1 , and the least poor in quintile 5 . This article focuses attention on body mass index (BMI) status in urban children in economically privileged (quintile 5) primary schools in Port Elizabeth. Quintile 5 children were assessed to infer a relationship between affluence and obesity prevalence in developing countries.

\section{Methods}

A descriptive one-way cross-sectional research design with baseline anthropometric measures of weight and height was used. ${ }^{[12]}$ The Nelson Mandela Metropolitan University (NMMU) Research Human Ethical Committee approved the study (H12-HEA-HMS-002).

\section{Sampling techniques}

A list of all the primary schools in the city of Port Elizabeth was obtained from the NMMU Department of Education. Of the total of 109 schools, 28 met the inclusion criteria of quintile 5, Englishmedium primary schools. Randomised sampling was used to select 10 schools from which to sample participants. Of the 10 schools selected, 4 were included in the study; only 4 of the principals gave permission for their learners to participate in the study. 


\section{Participants}

A total of 713 children in the 7 - 10-year-old age group were included as the target population. The rationale for inclusion of this age group was that body mass was unlikely to be affected by developmental changes associated with puberty or infancy. ${ }^{[9]}$ A demographic description pertaining to age, gender, ethnicity and home language of the participants is presented in Table 1.

\section{Data collection and fieldwork practice}

A week before the scheduled anthropometric data-gathering date, the class teacher provided participants with a booklet containing questions to be answered by parents or guardians regarding demographic information of age, gender, ethnicity and home language. Included were informed-consent and assent forms for children to be signed by the respective parties prior to voluntary participation. Anthropometric measures were documented in the booklet. Measurements were done in private classroom settings by trained postgraduate research assistants and completed within a 1-month period of appointment times. Procedures were explained to participants to reduce any uncertainty or anxiety. Children were measured according to school grade, and boys and girls were assessed separately.

\section{Measuring instruments and procedures}

The anthropometric instruments for weight and height were calibrated prior to measurement according to the respective manuals. Measurements were taken according to the standardised protocol of the American College of Sports Medicine. ${ }^{[13]}$

\section{Anthropometry}

Weight was measured using a Scalemaster RS-232C electronic scale, and the readings were noted to the nearest $0.01 \mathrm{~kg} .{ }^{[13]}$ The participants were tested while standing upright and motionless in the anatomical zero position, while looking at a fixed point at eye level in the Frankfurt plane. They were barefoot and wore minimal clothing. Body weight was distributed evenly on both feet.

Maximum height was measured using a Charder HM200PW stadiometer to the nearest $0.1 \mathrm{~cm} \cdot{ }^{[13]}$ Height was measured from the soles of the feet to the vertex of the head. Participants were instructed to stand upright with their heels, gluteal muscles, scapulae and heads touching the vertical surface of the stadiometer. Body weight was evenly distributed on both feet and arms were hanging relaxed by their sides, palms facing the thighs. The head was placed in the Frankfurt horizontal plane to ensure the vertex of the head was located. The movable block was placed on the vertex of the head and the examiner ensured the Frankfurt horizontal plane was kept at all times by holding onto the mandible while the participant inhaled maximally. Measurement of height was taken after full inhalation. No hair accessories were worn.

\section{BMI}

BMI was calculated from weight and height measurements $\left(\mathrm{kg} / \mathrm{m}^{2}\right)$. The internationally accepted normative International Obesity Task Force (IOTF) cut-off criteria (Table 3) were used to quantify obesity and overweight rates among participants. ${ }^{[14]}$ These criteria are based on and linked to the corresponding adult BMI cut-off values used as clinical measures of obesity. ${ }^{[14-16]}$ Most of the research evidence in SA, bar the Health of the Nation Study, used arbitrarily defined percentile cut-offs. ${ }^{[4,16]}$

\section{Statistical analysis}

Statistica version 9.0 was used for quantitative data analysis. ${ }^{[17]}$ Descriptive statistics were used to report on means and standard deviations (SDs) for age, gender and BMI status variables (using both normative percentiles and $z$-scores). One-way analysis of variance (ANOVA) was used to determine whether a difference in overweight and obesity levels existed for age and gender cohorts. ${ }^{[18]}$ Post-hoc analysis of results was done by means of $\chi^{2}$ tests. ${ }^{[18]}$ Significance was accepted at $p<0.05 .^{[18]}$

\section{Results}

The mean (SD) BMI scores for the total group $(N=713)$ is illustrated in Table 2. The maximum BMI score recorded was $31.6 \mathrm{~kg} / \mathrm{m}^{2}$ and the minimum was $12.6 \mathrm{~kg} / \mathrm{m}^{2}$. Converted BMI $z$-scores indicated a mean of $0.4(0.7)$; this was done in order to compare results to international normative data. ${ }^{[14]}$ The maximum BMI $z$-score achieved was 2 and

Table 1. Participant characteristics $(N=713)$

\begin{tabular}{lll}
\hline & \multicolumn{2}{c}{$\boldsymbol{n}$} \\
\cline { 2 - 3 } & Boys & Girls \\
\hline Age (years) & & \\
7 & 90 & 84 \\
8 & 81 & 86 \\
9 & 109 & 89 \\
10 & 92 & 82 \\
All groups & 372 & 341 \\
Ethnicity & & \\
White & 449 & \\
Black & 125 & \\
Mixed race & 91 & \\
Asian & 48 & \\
Home language & & \\
English & 547 & \\
Afrikaans & 54 & \\
isiXhosa & 108 \\
Other & 4 & \\
& &
\end{tabular}

Table 2. Descriptive statistics of BMI for the total group

\begin{tabular}{lll}
\hline Variable & $\boldsymbol{N}$ & Mean $(\mathrm{SD})$ \\
\hline BMI $\left(\mathrm{kg} / \mathrm{m}^{2}\right)$ & 713 & $18.4(3.2)$ \\
BMI (IOTF) $(z$-score) & 713 & $0.4(0.7)$ \\
$\quad$ Boys & 372 & $0.3(0.7)$ \\
$\quad$ Girls & 341 & $0.4(0.8)$ \\
Age (years, months) & 713 & $9.0(1.1)$ \\
BMI = body mass index; SD = standard deviation; IOTF = International Obesity Task Force.
\end{tabular}


the minimum was -3 . Boys and girls obtained average $z$-scores that placed them in the normal weight category when compared to internationally accepted IOTF $z$-score criteria for BMI classification (Table 3). ${ }^{[14]}$

Normative criteria ${ }^{[14]}$ and descriptive results (Table 3$)$ showed that $65.1 \%(n=464)$ of the total group were of normal weight, 20.9\% ( $n=149)$ were overweight and 9.8\% $(n=70)$ were obese. The highest prevalence of overweight $(24.7 \%, n=43, N=174)$ was found in children aged 10 and the highest prevalence of obesity $(12.5 \%, n=21, N=167)$ was found in 8-year-old children (Table 4). The lowest prevalence of overweight (18.5\%, $n=31)$ and obesity $(7.4 \%, n=13)$ were found in children aged 8 and 10, respectively. Posthoc $\chi^{2}$ test results revealed no significant interaction between BMI $z$-scores for age cohorts as indicated by $\chi^{2}=13.1, p>0.05$.

Results analysed for gender effect showed that $18.6 \%(n=69)$ of the boys $(N=372)$ were overweight and $6.9 \%(n=26)$ were obese
(Table 4). In comparison, 23.5\% ( $n=80$ ) of girls $(N=341)$ were overweight and $12.9 \%(n=44)$ were obese. ANOVA yielded a significant difference in BMI IOTF $z$-scores between boys and girls $(F=7.2, p<0.05)$.

\section{Discussion}

The prevalence of childhood obesity in developing countries is associated more with children from higher socioeconomic areas than their less economically privileged counterparts. ${ }^{[19]}$ Despite the worldwide evidence on the obesity epidemic, there is still a lack of literature on obesity rates among prepubertal urban children in SA from affluent higher socioeconomic areas. ${ }^{[2]}$

Findings from this study indicated that most participants were classified as normal or with optimal BMI according to international criteria for growing children. ${ }^{[14]}$ Overweight and obesity prevalence rates were $20.9 \%$ and $9.8 \%$, respectively. These percentages are consistent with statistics published by the Human

Table 3. BMI criteria (IOTF z-scores) and classification of total group

\begin{tabular}{lllll}
\hline & & \multicolumn{3}{c}{ IOTF $\boldsymbol{z}$-score } \\
\cline { 3 - 5 } Grade & BMI range at 18 years & $\boldsymbol{z}$-score & $\boldsymbol{n}$ & $\%$ \\
\hline Thinness grade 3 (TG3) & $<16$ & -3 & 1 & 0.1 \\
Thinness grade 2 (TG2) & $16-<17$ & -2 & 5 & 0.7 \\
Thinness grade 1 (TG1) & $17-<18.5$ & -1 & 24 & 3.4 \\
Normal weight (Norm) & $18.5-<25$ & 0 & 464 & 65.1 \\
Overweight (Ovw) & $25-<30$ & 1 & 149 & 20.9 \\
Obese (O) & $30+$ & 2 & 70 & 9.8 \\
All groups & & & 713 & 100 \\
BMI = body mass index; IOTF = International Obesity Task Force. & & &
\end{tabular}

Development Index (HDI) for childhood overweight and obesity prevalence in SA, namely $31.8 \%$ and $8.1 \%$, respectively. ${ }^{[4,20]}$ Comparative differences in prevalence rates reported both here and in the Health of the Nation Study ${ }^{[4]}$ could be attributed to the smaller sample size of this study and the inclusion of 7 - 10-year-olds from an affluent, economically privileged urban area. The national study surveyed 6 - 13-year-olds from all socioeconomic strata $(N=10195) \cdot{ }^{[4]}$

Challenges arose when making comparisons with SA studies, as only the Armstrong et al. ${ }^{[4]}$ study, which used the latest Cole et al. ${ }^{[14]}$ IOTF BMI grading system, could be found in the literature search. Inclusion criteria for selected sample populations differ across studies; this created further difficulties when contrasting the literature. Truter et al. ${ }^{[21]}$ analysed children aged $9-13$ years $(N=280)$ from various socioeconomic strata and indicated an overweight and obesity prevalence rate of $15.5 \%$ and $6.5 \%$, respectively. Kemp et al. ${ }^{[22]}$ analysed the BMI values of 7 -year-old children $(N=816)$ from lower socioeconomic areas and reported that $7.8 \%$ of the participants were overweight and $3.8 \%$ were obese. Both of these studies used previous methods of arbitrarily defined percentile cut-offs and smaller sample sizes with variation in variables. ${ }^{[21,22]}$ The perception currently exists that rural children from impoverished environments have lower prevalence of obesity and overweight than their counterparts with higher socioeconomic status; ${ }^{[19]}$ however, more scientific evidence, including studies with larger sample sizes, equivalent variables and the use

Table 4. BMI criteria (IOTF $z$-score) and classification by age group and gender

\begin{tabular}{|c|c|c|c|c|c|c|c|c|c|c|c|c|c|c|c|c|c|c|c|c|}
\hline \multirow{4}{*}{$\frac{\text { Age (year) }}{\text { Classification }}$} & \multicolumn{20}{|c|}{ IOTF $z$-score } \\
\hline & \multicolumn{4}{|c|}{7} & \multicolumn{4}{|c|}{8} & \multicolumn{4}{|c|}{9} & \multicolumn{4}{|c|}{10} & \multicolumn{4}{|c|}{ All groups } \\
\hline & \multicolumn{2}{|c|}{$n$} & \multicolumn{2}{|c|}{$\%$} & \multicolumn{2}{|c|}{$n$} & \multicolumn{2}{|c|}{$\%$} & \multicolumn{2}{|c|}{$n$} & \multicolumn{2}{|c|}{$\%$} & \multicolumn{2}{|c|}{$n$} & \multicolumn{2}{|c|}{$\%$} & \multicolumn{2}{|c|}{$n$} & \multicolumn{2}{|c|}{$\%$} \\
\hline & B & G & B & G & B & G & B & G & B & G & B & G & B & G & B & G & B & G & B & G \\
\hline TG3 & 0 & 0 & 0 & 0 & 0 & 0 & 0 & 0 & 0 & 0 & 0 & 0 & 0 & 1 & 0 & 1.2 & 0 & 1 & 0 & 0.3 \\
\hline TG2 & 0 & 0 & 0 & 0 & 1 & 1 & 1.2 & 1.2 & 0 & 1 & 0 & 1.1 & 1 & 1 & 1.1 & 1.2 & 2 & 3 & 0.5 & 0.9 \\
\hline TG1 & 2 & 1 & 2.2 & 1.2 & 5 & 3 & 6.2 & 3.5 & 2 & 5 & 1.8 & 5.6 & 3 & 3 & 3.3 & 3.7 & 12 & 12 & 3.2 & 3.5 \\
\hline Norm & 67 & 54 & 74.4 & 64.3 & 52 & 53 & 64.2 & 61.6 & 82 & 47 & 75.2 & 52.8 & 62 & 47 & 67.4 & 57.3 & 263 & 201 & 70.7 & 59.2 \\
\hline Ovw & 13 & 22 & 14.4 & 26.2 & 16 & 15 & 19.8 & 17.4 & 18 & 22 & 16.5 & 24.7 & 22 & 21 & 23.9 & 25.6 & 69 & 80 & 18.6 & 23.5 \\
\hline $\mathrm{O}$ & 8 & 7 & 8.9 & 8.3 & 7 & 14 & 8.6 & 16.3 & 7 & 14 & 6.4 & 15.7 & 4 & 9 & 4.4 & 11 & 26 & 44 & 6.9 & 12.9 \\
\hline All groups & 90 & 84 & 100 & 100 & 81 & 86 & 100 & 100 & 109 & 89 & 100 & 100 & 92 & 82 & 100 & 100 & 372 & 341 & 100 & 100 \\
\hline
\end{tabular}

$\mathrm{BMI}=$ body mass index; IOTF = International Obesity Task Force; B = boy; $\mathrm{G}=$ girl; TG = thinness grade; Norm = normal; Ovw = overweight; $\mathrm{O}=$ obese. 
of internationally accepted BMI criteria, are needed to confirm this trend in SA.

The HDI ${ }^{[20]}$ categorises SA as a country in transition, similar to Algeria, Egypt, China and India; therefore it is relevant to make a comparison with international studies. The $\mathrm{HDI}^{[20]}$ reported childhood prevalence rates of $24 \%$ overweight and $11.4 \%$ obesity in Egypt, $10.5 \%$ overweight and $7.9 \%$ obesity in Algeria, and $29.6 \%$ overweight and $9.3 \%$ obesity in India. Westernisation of diet and the availability of inexpensive foods of poor nutritious quality have been linked to increased obesity incidence in children. ${ }^{[23]}$ Although Kruger et al. ${ }^{[23]}$ found no significant relationship between obesity and the effects of urbanisation, a pattern emerged that showed that urban-dwelling children consumed more calories and had the highest BMI values compared with rural children. These findings were confirmed by data from the SA National Food Consumption survey, which found that the highest prevalence of overweight children was in urban areas. ${ }^{[23]}$

Consistent with the literature, ${ }^{[4,11]}$ significant gender effects were found in the study where girls had higher overweight and obesity rates than boys. Of concern is the finding that girls had almost double the obesity rates of boys. This prevalence is higher than data reported in research mentioned earlier. ${ }^{[2-4]}$ Possible reasons for gender differences could be lower physical activity levels in girls, ${ }^{[24]}$ and biological and sociocultural differences. ${ }^{[25]}$

\section{Conclusions}

The prevalence of overweight and obesity among preadolescent urban children in an affluent geographic setting was high. Children as young as 8 years were found to be obese, more so than 10-yearolds who were overweight rather than obese. This trend is alarming when compared with earlier studies, as young children in this study showed higher BMI levels at an earlier age than previously documented. Girls displayed almost double the occurrence of obesity in boys. This growing obesity incidence has escalated rapidly since published results in 2002 in the SA Youth Risk Behaviour Survey.

In the light of these findings, further research is needed on regional and national patterns in body mass status in young children from all socioeconomic strata, ethnicities, cultures and geographical areas in SA. Beyond individual characteristics, the built environment (access to recreational and sport facilities near schools, access to destinations and public transportation, presence of pavements and controlled intersections, the proportion of green space available for active play) has an impact on obesogenic behaviour, and future studies should identify and address the need for developmentally appropriate, focused interventions for specific childhood populations. The recent internationally standardised method of assessment is more appropriate for children younger than 18 years and should be used for comparisons and to monitor secular trends in obesity among children. Future studies could expand on the current use of crosssectional experimental designs by doing longitudinal research to guide interventions and educational policies more effectively.

The implication for health professionals is to be aware of the modifiable causes of childhood obesity and to implement physical activity, exercise and nutrition management as a primary approach to intervention.

Acknowledgements. Financial assistance was received from the National Research Foundation and the NMMU as a research grant.

\section{References}

1. World Health Organization. Obesity and Overweight - Fact Sheet Number 311. Geneva: World Health Organization, 2013:1.

2. Rossouw H, Grant C, Viljoen M. Overweight and obesity in children and adolescents: The SA problems. S Afr J Sci 2012;108(5/6):907-914. [http://dx.doi.org/10.4102/sajs.v108i5/6.907]

3. Reddy S, Panday S, Swart D, et al. Umthenethe Uhlaba Usamila: The South African Youth Risk Behaviour Survey, 2002. Cape Town: South African Medical Research Council, 2003.

4. Armstrong M, Lambert M, Sharwood K, Lambert E. Obesity and overweight in South African primary school children: The Health of the Nation Study. S Afr Med J 2006;96(5):439-444

5. Li C, Ford E, Huang T, Sun S, Goodman E. Patterns of change in cardiometabolic risk factors associated with the metabolic syndrome among children and adolescents: The Fels longitudinal study. J Paediatr 2009;155(3):S5.e9-S9,e16. [http://dx.doi. org/10.1016/j.jpeds.2009.04.046]

6. Binkeiwicz-Glinska A, Bakula S, Kusiak-Kaczmarek M, et al. Obesity prevention in children and adolescents: Current recommendations. Pol Ann Med 2012 July;19(2):158162. [htt[://dx.doi.org/10.1016/j.poamed.2012.07.003]

7. Kelishadi R. Childhood overweight, obesity and the metabolic syndrome in developing countries. Epidemiol Rev 2007; 29(1):62-76. [http://dx.doi.org/10.1093/epirev/mxm003]

8. World Health Organization. Growth Reference 5 - 19 years: Age-for-height and BMI. Geneva: World Health Organization, 2007.

9. Santrock J. Child Development. 11th ed. USA: McGraw-Hill, 2005:165.

10. Monyeki K, Van Lenthe K, Steyn N. Obesity: Does it occur in African children in a rural community of South Africa? Int J Epidemiol 1999; 28(2):287-292.

11. Jacobs S, De Ridder H. Prevalence of overweight and underweight among black South African children from rural areas in the North-West Province. South African Journal for Research in Sport, Physical Education and Recreation 2012;34(2):41-51.

12. Carter R, Lubinsky J, Domholdt E. Rehabilitation Research: Principles and Applications. 4th ed. USA: Elsevier Saunders, 2011:56-58.

13. American College of Sports Medicine. ACSM's Guidelines for Exercise Testing and Prescription. 8th ed. London: Lippincott, Williams and Wilkins, 2010.

14. Cole T, Flegal K, Nicholls D, Jackson A. Body mass index cut offs to define thinness in children and adolescents: International survey. BMJ 2007;335:194-197. [http://dx.doi. org/10.1136/bmj.39238.399444.55]

15. August G, Caprio S, Fennoy H, et al. Prevention and treatment of paediatric obesity: An endocrine society clinical practice guideline based on expert opinion. J Clin Endocrinol Metab 2008;93(12):4576-4599. [http://dx.doi.org/10.1210/jc.2007-2458]

16. Armstrong $M$, Lambert M, Lambert E. Secular trends in the prevalence of stunting, overweight and obesity among South African children (1994 - 2004). Eur J Clin Nutr 2011;65(7): 835-840. [http://dx.doi.org/10.1038/ejcn.2011.46]

17. Statsoft. Statistica for Windows. 8th ed. USA: StatSoft, 2008.

18. Mendenhall W, Beaver R, Beaver B. Introduction to Probability and Statistics. 14th ed. Boston, USA: Brooks/Cole Cengage Learning, 2013.

19. Raj M, Kumar K. Obesity in children and adolescents. Indian J Med Res 2010;132(1):598-607.

20. United Nations Development Programme. Human Development Index (HDI). http:// hdr.undp.org/en/statistics/ (accessed on 9 October 2013).

21. Truter L, Pienaar A, Du Toit D. Relationships between overweight, obesity and physical fitness of nine- to-twelve-year-old South African children. South African Family Practice 2010:52(3):227-233.

22. Kemp C, Pienaar A, Schutte A. The prevalence of hypertension and the relationship with body composition in Grade 1 learners in the North-West Province of South Africa. South African Journal of Sports Medicine 2011;23(4):117-122. [http://dx.doi.org/10.7196/ sajsm.244]

23. Kruger R, Kruger H, MacIntyre U. The determinants of overweight and obesity among 10- to 15-year-old school children in the North-West Province, South Africa: The THUSA BANA study. Public Health Nutr 2006;9(3):351-358. [http://dx.doi. org/10.1079/PHN2005849]

24. Ortlieb S, Schneider G, Koletzko S, et al. Physical activity and its correlates in children: A cross-sectional study (the GINIplus and LISAplus studies). BMC Public Health 2013;13:349-363. [http://dx.doi.org/10.1186/1471-2458-13-349]

25. Sweeting H. Gendered dimensions of obesity in childhood and adolescence. Nutr J 2008;7(1):1-14. [http://dx.doi.org/10.1186/1475-2891-7-1] 